\title{
Multilinear commutators of fractional integrals over Morrey spaces with non-doubling measures
}

\author{
Xiangxing Tao and Taotao Zheng
}

\begin{abstract}
In this paper, the authors study the boundedness of multilinear fractional integrals on the product Morrey space with non-doubling measure, and investigate the Morrey boundedness properties of the multilinear commutators generated by multilinear fractional integral operators with a tuple of $R B M O$ functions.
\end{abstract}

Mathematics Subject Classification (2010). 42B20, 42B25, 42B30.

Keywords. Multi-commutator, Multilinear fractional integral, $R B M O$ function, Morrey space, Non-doubling measure.

\section{Introduction and main results}

The classical result of commutator is due to Coifman et al. [1]: if $b \in B M O$ and $T$ is a Calderón-Zygmund operator, then the commutator $[b, T]$ is bounded on $L^{p}$ spaces for $1<p<\infty$. In 2001, Tolsa [2] developed the theory of CalderónZygmund operators and their commutators with $R B M O$ functions in the setting of non-doubling measures. Later on, Chen and Sawyer [3] modified the definition of $R B M O$ to investigate the commutators of the potential operators and $R B M O$ functions. Recently, Sawano and Tanaka [4,5] defined the Morrey spaces for non-doubling measures, and obtained the boundedness of the commutators generated by fractional integrals with $R B M O$ functions in Morrey spaces. Here we point out that the commutator estimates for non-doubling measures play an important role in solving the long-standing open questions, like the Painlevè problem.

In recent years, the theory on multilinear integral operators and multilinear commutators have attracted much attentions as a rapid developing field in

This work was supported in part by the NNSF of China under Grant \#10771110 and \#10471069, and sponsored by the NSF of Ningbo City under Grant \#2009A610090. 
harmonic analysis. Hu et al. [6] studied the $L^{p}$-boundedness and certain weak type endpoint estimates for multilinear commutators generated by multilinear Calderón-Zygmund singular integral with $R B M O$ functions. In this paper, we will extend the works of Sawano and Tanaka $[4,5,7]$ and Tao et al. [8] to the context for multilinear commutators generated by multilinear fractional integrals and $R B M O$ functions over the Morrey spaces with non-doubling measures.

Throughout this paper $\mu$ will be a positive Radon measures on $\mathbb{R}^{d}$ satisfing the growth condition: there exist a constant $C>0$ and $n \in(0, d]$ such that

$$
\mu(Q) \leq C l(Q)^{n}
$$

for any cube $Q \in \mathbb{R}^{d}$ with sides parallel to the coordinate axes. $Q(x, l(Q))$ will be the cube centered at $x$ with side length $l(Q)$. For $r>0, r Q$ will denote the cube with the same center as $Q$ and with $l(r Q)=r l(Q)$. The set of all cubes $Q \subset \mathbb{R}^{d}$ satisfying $\mu(Q)>0$ is denoted by $\mathscr{Q}(\mu)$.

Before stating our main results, we fix some notations and define some terminologies. Given $\beta_{d} \geq 2^{d+1}$ large enough but depending only on the dimension $d$, we say that a cube $Q \subset \mathbb{R}^{d}$ is doubling if $\mu(2 Q) \leq \beta_{d} \mu(Q)$. For any fixed cube $Q \subset \mathbb{R}^{d}$, let $N \geq 0$ be the smallest integer such that $2^{N} Q$ is doubling. We denote this cube by $\widetilde{Q}$.

Let $0 \leq \alpha<n$, for two cubes $Q \subset R$ in $\mathbb{R}^{d}$, we always set

$$
K_{Q, R}^{(\alpha)}=1+\sum_{k=1}^{N_{Q, R}}\left[\frac{\mu\left(2^{k} Q\right)}{l\left(2^{k} Q\right)^{n}}\right]^{1-\frac{\alpha}{m n}},
$$

where $N_{Q, R}$ is the first positive integer $k$ such that $l\left(2^{k} Q\right) \geq l(R)$. If $\alpha=0$, then $K_{Q, R}^{(\alpha)}=K_{Q, R}$, The latter was introduced by Tolsa in [2].

Denote by $m_{Q} f$ the mean value of $f$ on $Q$, namely, $m_{Q} f=\frac{1}{\mu(Q)} \int_{Q} f(x) d \mu$. Let $\eta>1$ be a fixed constant, we say that $f \in L_{l o c}^{1}(\mu)$ is in $R B M O(\mu)$ if there exist a constant $\mathcal{A}$ such that

$$
\frac{1}{\mu(\eta Q)} \int_{Q}\left|f(y)-m_{\widetilde{Q}} f\right| d \mu(y) \leq \mathcal{A}
$$

for any cube $Q$, and

$$
\left|m_{Q} f-m_{R} f\right| \leq \mathcal{A} K_{Q, R}
$$

for any two doubling cubes $Q \subset R$. The minimal constant $\mathcal{A}$ is the $R B M O(\mu)$ norm of $f$, and it will be denoted by $\|f\|_{*}$.

We recall the definition of the Morrey space with non-doubling measure.

Definition 1.1. Let $k>1$, and $1 \leq q \leq p<\infty$, the Morrey space $M_{q}^{p}(k, \mu)$ is defined as

$$
M_{q}^{p}(k, \mu):=\left\{f \in L_{l o c}^{q}(\mu) ;\|f\|_{M_{q}^{p}(k, \mu)}<\infty\right\}
$$


with the norm

$$
\|f\|_{M_{q}^{p}(k, \mu)}:=\sup _{Q \in \mathscr{Q}(\mu)} \mu(k Q)^{\frac{1}{p}-\frac{1}{q}}\left(\int_{Q}|f|^{q} d \mu\right)^{\frac{1}{q}} .
$$

As is easily seen, the space $M_{q}^{p}(k, \mu)$ is a Banach space with its norm. The Morrey space norm reflects local regularity of $f$ more precisely than the Lebesgue space norm. It is easy to see from the Hölder inequality that $L^{p}(\mu)=$ $M_{p}^{p}(k, \mu) \subset M_{q_{1}}^{p}(k, \mu) \subset M_{q_{2}}^{p}(k, \mu)$ whenever $1 \leq q_{2} \leq q_{1} \leq p<\infty$. Moreover, the definition of the spaces is independent of the constant $k>1$, and the norms for different choice of $k>1$ are equivalent, see $[4,5,7]$ for details. We will denote $M_{q}^{p}(2, \mu)$ by $M_{q}^{p}(\mu)$.

Denoting by $\vec{f}=\left(f_{1}, f_{2}, \ldots, f_{m}\right)$, we consider in this article the multilinear fractional integral operator $I_{\alpha, m}$ as follows,

$I_{\alpha, m}(\vec{f})(x)=\int_{\left(\mathbb{R}^{d}\right)^{m}} \frac{f_{1}\left(y_{1}\right) \cdots f_{m}\left(y_{m}\right) d \mu\left(y_{1}\right) \cdots d \mu\left(y_{m}\right)}{\left|\left(x-y_{1}, x-y_{2}, \ldots, x-y_{m}\right)\right|^{m n-\alpha}} \quad$ for $x \in \mathbb{R}^{d}$.

Let $b_{i} \in R B M O(\mu)$ for $i=1,2, \ldots, m$, and let $\vec{b}=\left(b_{1}, b_{2}, \ldots, b_{m}\right)$, then the multilinear commutator $\left[\vec{b}, I_{\alpha, m}\right]$ is formally defined as

$$
\left[\vec{b}, I_{\alpha, m}\right](\vec{f})(x)=\int_{\left(\mathbb{R}^{d}\right)^{m}} \prod_{j=1}^{m}\left(b_{j}(x)-b_{j}\left(y_{j}\right)\right) \frac{f_{1}\left(y_{1}\right) \cdots f_{m}\left(y_{m}\right) d \mu\left(y_{1}\right) \cdots d \mu\left(y_{m}\right)}{\left|\left(x-y_{1}, x-y_{2}, \ldots, x-y_{m}\right)\right|^{m n-\alpha}} .
$$

More generally, denote by $C_{i}^{m}$ the family of all subsets $\sigma=\left\{\sigma_{1}, \sigma_{2}, \ldots, \sigma_{i}\right\}$ of $i$ different elements of $\{1,2, \ldots, m\}$, and let $\sigma^{\prime}=\{1,2, \ldots, m\} \backslash \sigma$ and $\overrightarrow{b_{\sigma}}=$ $\left\{b_{\sigma_{1}}, b_{\sigma_{2}}, \ldots, b_{\sigma_{i}}\right\}$. For any $\sigma \in C_{i}^{m}$, we define

$$
\begin{aligned}
{\left[\overrightarrow{b_{\sigma}}, I_{\alpha, m}\right](\vec{f})(x)=} & \int_{\left(\mathbb{R}^{d}\right)^{m}} \prod_{\sigma_{j} \in \sigma}\left(b_{\sigma_{j}}(x)-b_{\sigma_{j}}\left(y_{j}\right)\right) \\
& \times \frac{f_{1}\left(y_{1}\right) \cdots f_{m}\left(y_{m}\right) d \mu\left(y_{1}\right) \cdots d \mu\left(y_{m}\right)}{\left|\left(x-y_{1}, x-y_{2}, \ldots, x-y_{m}\right)\right|^{m n-\alpha}} .
\end{aligned}
$$

In case $\sigma=\{1,2, \ldots, m\}$, one sees that $\left[\overrightarrow{b_{\sigma}}, I_{\alpha, m}\right]$ is just the commutator $\left[\vec{b}, I_{\alpha, m}\right]$.

Here we remark that the multilinear commutator $\left[\vec{b}, I_{\alpha, m}\right]$ can be equivalently rewritten as

$$
\left[\vec{b}, I_{\alpha, m}\right](\vec{f})(x)=\left[b_{m},[b_{m-1}, \ldots,\left[b_{1}, I_{\alpha, m}\right] \overbrace{\cdots \cdot]}^{m}(\vec{f})(x) .\right.
$$

The main results in this paper can be stated as follows.

Theorem 1.2. Suppose that $0<\alpha<m n$ and $1<q_{i} \leq p_{i}<\infty$, and let $\frac{1}{p}=\frac{1}{p_{1}}+\frac{1}{p_{2}}+\cdots+\frac{1}{p_{m}}-\frac{\alpha}{n}>0, \frac{1}{q}=\frac{1}{q_{1}}+\frac{1}{q_{2}}+\cdots+\frac{1}{q_{m}}-\frac{\alpha}{n}>0$, then

$$
\left\|I_{\alpha, m}(\vec{f})\right\|_{M_{q}^{p}(\mu)} \leq C \prod_{i=1}^{m}\left\|f_{i}\right\|_{M_{q_{i}}^{p_{i}}(\mu)}
$$

with the constant $C$ independent of $\vec{f}$. 
Remark 1.3. In the case $p_{i}=q_{i}$, Theorem 1.2 implies the $L^{p}$-boundedness for the operator $I_{\alpha, m}$, precisely,

$$
\left\|I_{\alpha, m}(\vec{f})\right\|_{L^{p}(\mu)} \leq C \prod_{i=1}^{m}\left\|f_{i}\right\|_{L^{p_{i}}(\mu)}
$$

with $\frac{1}{p}=\frac{1}{p_{1}}+\frac{1}{p_{2}}+\cdots+\frac{1}{p_{m}}-\frac{\alpha}{n}>0$ for any $1<p_{i}<\infty$.

Theorem 1.4. Let $0<\alpha<m n$ and $1<q_{i} \leq p_{i}<\infty$, and let $\frac{1}{p}=\frac{1}{p_{1}}+\frac{1}{p_{2}}+$ $\cdots+\frac{1}{p_{m}}-\frac{\alpha}{n}>0, \frac{1}{q}=\frac{1}{q_{1}}+\frac{1}{q_{2}}+\cdots+\frac{1}{q_{m}}-\frac{\alpha}{n}>0$. Suppose $\|\mu\|=\infty$, and that $b_{i} \in R B M O(\mu)$ for $i=1,2, \ldots, m$. Then, for all $\sigma \subseteq\{1,2, \ldots, m\}$, the commutators $\left[\vec{b}_{\sigma}, I_{\alpha, m}\right]$ are bounded from $M_{q_{1}}^{p_{1}}(\mu) \times M_{q_{2}}^{p_{2}}(\mu) \times \cdots \times M_{q_{1}}^{p_{1}}(\mu)$ to $M_{q}^{p}(\mu)$, moreover,

$$
\left\|\left[\vec{b}_{\sigma}, I_{\alpha, m}\right](\vec{f})\right\|_{M_{q}^{p}(\mu)} \leq C \prod_{j \in \sigma}\left\|b_{j}\right\|_{*} \prod_{i=1}^{m}\left\|f_{i}\right\|_{M_{q_{i}}^{p_{i}}(\mu)},
$$

and particularly,

$$
\left\|\left[\vec{b}, I_{\alpha, m}\right](\vec{f})\right\|_{M_{q}^{p}(\mu)} \leq C \prod_{i=1}^{m}\left\|b_{i}\right\|_{*}\left\|f_{i}\right\|_{M_{q_{i}}^{p_{i}(\mu)}},
$$

where $C>0$ is the constant independent of $\vec{f}, \vec{b}$ and $\vec{b}_{\sigma}$.

Remark 1.5. In the case $m=1$, Theorem 1.2 and 1.4 follow that the fractional integral operator $I_{\alpha}$ and its commutator $\left[b, I_{\alpha}\right]$ are all bounded from $M_{q_{1}}^{p_{1}}(\mu)$ to $M_{q}^{p}(\mu)$, which are just Theorem 3.3 of [4] and Theorem 4.6 of [5], so our theorems contain their conclusions. In [8] the authors obtained the same result as Theorem 1.2 if $\mu$ is the Lebesgue measure.

To show Theorem 1.2 in Sect. 2, we will use a maximal function $M_{k} f(x)$ and give a pointwise estimate for the multilinear fractional integral $I_{\alpha, m}(\vec{f})(x)$ in the setting of non-doubling measures. In order to derive Theorem 1.4, we will exploit the decomposition technique for the product of functions and overcome the difficult brought from the multilinear operators and non-doubling measures. We will establish a pointwise estimate for the sharp maximal function of the commutator $\left[\vec{b}, I_{\alpha, m}\right](\vec{f})$ by using the non-centered maximal operator $M_{\tau, \eta}^{(\alpha)}$, see Lemma 3.4 below. We will prove Theorem 1.4 in Sect. 3.

\section{The proof of Theorem 1.2}

Before proving the theorem, we recall the following maximal operator,

$$
M_{k} f(x)=\sup _{x \in Q \subset \mathscr{Q}(\mu)} \frac{1}{\mu(k Q)} \int_{Q}|f(y)| d \mu(y),
$$

and some lemmas which will be used in this paper. 
Lemma 2.1. [4] If $1<q \leq p<\infty$, then there exist a constant $C$ independent of $f$ such that

$$
\left\|M_{k} f\right\|_{M_{q}^{p}(\mu)} \leq C\|f\|_{M_{q}^{p}(\mu)} .
$$

Lemma 2.2. For $0<\alpha<m n, 1<q_{i} \leq p_{i}<\infty, \frac{1}{h}=\frac{1}{p_{1}}+\frac{1}{p_{2}}+\cdots+\frac{1}{p_{m}}$, $\frac{1}{p}=\frac{1}{h}-\frac{\alpha}{n}>0$, we have

$$
\left|I_{\alpha, m} \vec{f}(x)\right| \leq C\left(\prod_{i=1}^{m}\left\|f_{i}\right\|_{M_{q_{i}}^{p_{i}}(\mu)}\right)^{1-\frac{h}{p}}\left(\prod_{i=1}^{m} M_{k} f_{i}(x)\right)^{\frac{h}{p}}
$$

with the absolute constant $C$ independent of $\vec{f}$.

Proof. For $x, y_{i} \in \mathbb{R}^{d}$ and $x \neq y_{i}, i=1,2, \ldots, m$, we can see from the Fubini theorem that

$$
\begin{aligned}
I_{\alpha, m}(\vec{f})(x) & \leq \int_{\left(\mathbb{R}^{d}\right)^{m}} \frac{\left|f_{1}\left(y_{1}\right)\right|\left|f_{2}\left(y_{2}\right)\right| \cdots\left|f_{m}\left(y_{m}\right)\right| d \mu\left(y_{1}\right) d \mu\left(y_{2}\right) \cdots d \mu\left(y_{m}\right)}{\left|\left(x-y_{1}, x-y_{2}, \ldots, x-y_{m}\right)\right|^{m n-\alpha}} \\
& \leq C \int_{\left(\mathbb{R}^{d}\right)^{m}} \int_{\left|\left(x-y_{1}, x-y_{2}, \ldots, x-y_{m}\right)\right|}^{\infty} l^{\alpha-m n-1} d l\left(\prod_{i=1}^{m}\left|f_{i}\left(y_{i}\right)\right| d \mu\left(y_{i}\right)\right) \\
& \leq C \int_{0}^{\infty}\left(\prod_{i=1}^{m} \frac{1}{l^{n}} \int_{\left|\left(x-y_{i}\right)\right|<l}\left|f_{i}\left(y_{i}\right)\right| d \mu\left(y_{i}\right)\right) l^{\alpha-1} d l .
\end{aligned}
$$

Using the Hölder inequality and the growth condition (1.1) for the nondoubling measure $\mu$, and letting cube $Q=Q(x, 2 l)$, then we have

$$
\begin{aligned}
& \int_{0}^{\lambda}\left(\prod_{i=1}^{m} \frac{1}{l^{n}} \int_{\left|\left(x-y_{i}\right)\right|<l}\left|f_{i}\left(y_{i}\right)\right| d \mu\left(y_{i}\right)\right) l^{\alpha-1} d l \\
& \leq C \int_{0}^{\lambda}\left(\prod_{i=1}^{m} \frac{1}{\mu(k Q)} \int_{Q}\left|f_{i}\left(y_{i}\right)\right| d \mu\left(y_{i}\right)\right) l^{\alpha-1} d l \\
& \leq C \int_{0}^{\lambda}\left(\prod_{i=1}^{m} M_{k} f_{i}(x)\right) l^{\alpha-1} d l \leq C \lambda^{\alpha} \prod_{i=1}^{m} M_{k} f_{i}(x) .
\end{aligned}
$$

On the other hand, with the similar ways as above we have

$$
\begin{aligned}
\frac{1}{l^{n}} \int_{\left|\left(x-y_{i}\right)\right|<l}\left|f_{i}\left(y_{i}\right)\right| d \mu\left(y_{i}\right) & \leq \frac{C}{l^{n}} \int_{Q}\left|f_{i}\left(y_{i}\right)\right| d \mu\left(y_{i}\right) \\
& \leq \frac{C}{l^{n}}\left(\int_{Q}\left|f_{i}\left(y_{i}\right)\right|^{q_{i}} d \mu\left(y_{i}\right)\right)^{\frac{1}{q_{i}}} \mu(Q)^{1-\frac{1}{q_{i}}} \\
& \leq C l^{-\frac{n}{p_{i}}}\left\|f_{i}\right\|_{M_{q_{i}}^{p_{i}}(\mu)}
\end{aligned}
$$


which means that

$$
\begin{aligned}
& \int_{\lambda}^{\infty}\left(\prod_{i=1}^{m} \frac{1}{l^{n}} \int_{\left|\left(x-y_{i}\right)\right|<l}\left|f_{i}\left(y_{i}\right)\right| d \mu\left(y_{i}\right)\right) l^{\alpha-1} d l \\
& \quad \leq C \int_{\lambda}^{\infty}\left(\prod_{i=1}^{m} l^{-\frac{n}{p_{i}}}\left\|f_{i}\right\|_{M_{q_{i}}^{p_{i}}(\mu)}\right) l^{\alpha-1} d l \leq C \lambda^{-\frac{n}{p}} \prod_{i=1}^{m}\left\|f_{i}\right\|_{M_{q_{i}}^{p_{i}}(\mu)} .
\end{aligned}
$$

Thus, by (2.1), we obtain that

$$
\left|I_{\alpha, m} \vec{f}(x)\right| \leq C\left[\lambda^{\alpha} \prod_{i=1}^{m} M_{k} f_{i}(x)+\lambda^{-\frac{n}{p}} \prod_{i=1}^{m}\left\|f_{i}\right\|_{M_{q_{i}}^{p_{i}}(\mu)}\right] .
$$

This yields the lemma by choosing the suitable positive real number $\lambda$.

Now we give the proof of theorem 1.2. Note $0<\alpha<m n, 1<q_{i} \leq p_{i}<$ $\infty, \frac{1}{h}=\frac{1}{p_{1}}+\frac{1}{p_{2}}+\cdots+\frac{1}{p_{m}}$, and let $\frac{1}{r}=\frac{1}{q_{1}}+\frac{1}{q_{2}}+\cdots+\frac{1}{q_{m}}$. By the lemma 2.2, one can get that

$$
\begin{aligned}
\left\|I_{\alpha, m} \vec{f}\right\|_{M_{q}^{p}(\mu)} \leq & C\left(\prod_{i=1}^{m}\left\|f_{i}\right\|_{M_{q_{i}}^{p_{i}(\mu)}}\right)^{1-\frac{h}{p}} \\
& \times \sup _{Q \in \mathcal{Q}(\mu)}|\mu(k Q)|^{\frac{1}{p}-\frac{1}{q}}\left\{\int_{Q}\left(\prod_{i=1}^{m} M_{k} f_{i}(y)\right)^{\frac{q h}{p}} d \mu(y)\right\}^{\frac{1}{q}} .
\end{aligned}
$$

Since $\frac{p r}{q h}=\frac{n-r \alpha}{n-h \alpha}>1$, hence by the Hölder inequality and Lemma 2.1 it follows

$$
\begin{aligned}
& |\mu(k Q)|^{\frac{1}{p}-\frac{1}{q}}\left\{\int_{Q}\left(\prod_{i=1}^{m} M_{k} f_{i}(y)\right)^{\frac{q h}{p}} d \mu(y)\right\}^{\frac{1}{q}} \\
& \leq C|\mu(k Q)|^{\frac{1}{p}-\frac{h}{p r}}\left\{\int_{Q}\left(\prod_{i=1}^{m} M_{k} f_{i}(y)\right)^{r} d \mu(y)\right\}^{\frac{h}{p r}} \\
& \leq C\left\{|\mu(k Q)|^{\frac{1}{h}-\frac{1}{r}}\left\{\int_{Q}\left(\prod_{i=1}^{m} M_{k} f_{i}(y)\right)^{r} d \mu(y)\right\}^{\frac{1}{r}}\right\}^{\frac{h}{p}} \\
& \leq C\left\{\prod_{i=1}^{m}|\mu(k Q)|^{\frac{1}{p_{i}}-\frac{1}{q_{i}}}\left(\int_{Q}\left(M_{k} f_{i}(y)\right)^{q_{i}} d \mu(y)\right)^{\frac{1}{q_{i}}}\right\}^{\frac{h}{p}} \\
& \leq C\left(\prod_{i=1}^{m}\left\|M_{k} f_{i}\right\|_{M_{q_{i}}^{p_{i}}(\mu)}\right)^{\frac{h}{p}} \leq C\left(\prod_{i=1}^{m}\left\|f_{i}\right\|_{M_{q_{i}}^{p_{i}}(\mu)}\right)^{\frac{h}{p}} .
\end{aligned}
$$

Thus the theorem is obtained. 


\section{The proof of Theorem 1.4}

In this section we will use the sharp maximal estimates. Let $f$ be a function in $L_{l o c}^{1}(\mu)$, the sharp maximal function of $f$ is defined by

$$
\begin{aligned}
M^{\sharp,(\alpha)} f(x)= & \sup _{Q \ni x} \frac{1}{\mu\left(\frac{3}{2} Q\right)} \int_{Q}\left|f(y)-m_{\widetilde{Q}} f\right| d \mu(y) \\
& +\sup _{R \supset Q \ni x Q, R \text { doubling }} \frac{\left|m_{Q} f-m_{R} f\right|}{K_{Q, R}^{(\alpha)}} .
\end{aligned}
$$

The non-centered doubling maximal operator is defined by

$$
N f(x)=\sup _{\substack{Q \ni x \\ Q \text { doubling }}} \frac{1}{\mu(Q)} \int_{Q}|f(y)| d \mu(y) .
$$

By the Lebesgue differential theorem, it is easy to see that $|f(x)| \leq N f(x)$ for any $f \in L_{l o c}^{1}(\mu)$ and $\mu$-a.e. $x \in \mathbb{R}^{d}$. Define the non-centered maximal operator,

$$
M_{\tau, \eta}^{(\alpha)} f(x)=\sup _{Q \ni x}\left(\frac{1}{\mu(\eta Q)^{1-\frac{\tau \alpha}{n}}} \int_{Q}|f(y)|^{\tau} d \mu(y)\right)^{\frac{1}{\tau}}
$$

for $\eta>1$ and $\tau>1$, where the supremum is taking over all the cubes $Q$ containing the point $x$. Usually we denote $M^{\sharp,(0)}(f)$ simply by $M^{\sharp}(f)$ and $M_{\tau, \eta}^{(0)}(f)$ by $M_{\tau, \eta}(f)$.

From the work in [2], we know that $M_{\tau, \eta}$ is bounded on $L^{q}(\mu)$ for $q>$ $\tau>1$ and $\eta>1$. Moreover, we have the following lemma.

Lemma 3.1. Let $q>\tau>1, \eta>1$, and $1<q \leq p<\infty$, then the operator $M_{\tau, \eta}$ is bounded on $M_{q}^{p}(\mu)$ and

$$
\left\|M_{\tau, \eta}(f)\right\|_{M_{q}^{p}(\mu)} \leq C\|f\|_{M_{q}^{p}(\mu)}
$$

with the constant $C$ independent of $f$.

Proof. The lemma could be proved along the similar lines as that of Theorem 2.3 in [4], i.e. Lemma 2.1 of this paper. Fix a cube $Q_{0} \in \mathscr{Q}(\mu)$ and put $L=\frac{l\left(Q_{0}\right)}{2}$, and let $f_{1}=\chi_{\frac{\eta+7}{\eta-1} Q_{0}} f$ and $f_{2}=f-f_{1}$, then we have for all $y \in Q_{0}$ that

$$
M_{\tau, \eta} f(y) \leq M_{\tau, \eta} f_{1}(y)+M_{\tau, \eta} f_{2}(y)
$$

Noting for $y \in Q_{0}$,

$$
\begin{aligned}
M_{\tau, \eta} f_{2}(y) & \leq \sup _{\substack{y \in Q \in \mathscr{Q}(\mu) \\
l(Q) \geq \frac{8 L}{\eta-1}}}\left\{\frac{1}{\mu(\eta Q)} \int_{Q}|f|^{\tau} d \mu\right\}^{\frac{1}{\tau}} \\
& \leq \sup _{\substack{Q_{0} \subset Q \in \mathscr{Q}(\mu) \\
\mu\left(\frac{2 \eta}{1+\eta} Q\right)}}\left\{\frac{1}{Q}|f|^{\tau} d \mu\right\}^{\frac{1}{\tau}}
\end{aligned}
$$


because of the fact that $y \in Q_{0} \bigcap Q$ and $l(Q) \geq \frac{8 L}{(\eta-1)}$ yields $Q_{0} \subset \frac{1+\eta}{2} Q$. Thus, from the inequalities (3.4) and (3.5), the $L^{q}(\mu)$-boundedness for $M_{\tau, \eta}$, and the Hölder inequality we get that

$$
\begin{aligned}
& \mu\left(\frac{2 \eta(\eta+7)}{\eta^{2}-1} Q_{0}\right)^{\frac{1}{p}-\frac{1}{q}}\left(\int_{Q_{0}}\left(M_{\tau, \eta} f\right)^{q} d \mu\right)^{\frac{1}{q}} \\
& \leq \mu\left(\frac{2 \eta(\eta+7)}{\eta^{2}-1} Q_{0}\right)^{\frac{1}{p}-\frac{1}{q}}\left(\int_{Q_{0}}\left(M_{\tau, \eta} f_{1}\right)^{q} d \mu\right)^{\frac{1}{q}} \\
& \quad+\mu\left(Q_{0}\right)^{\frac{1}{p}-\frac{1}{q}}\left(\int_{Q_{0}}\left(M_{\tau, \eta} f_{2}\right)^{q} d \mu\right)^{\frac{1}{q}} \\
& \leq \mu\left(\frac{2 \eta(\eta+7)}{\eta^{2}-1} Q_{0}\right)^{\frac{1}{p}-\frac{1}{q}}\left(\int_{\frac{\eta+7}{\eta-1} Q_{0}}|f|^{q} d \mu\right)^{\frac{1}{q}} \\
& \quad+\sup _{Q_{0} \subset Q \in \mathscr{Q}(\mu)} \frac{\mu\left(Q_{0}\right)^{\frac{1}{p}}}{\mu\left(\frac{2 \eta}{1+\eta} Q\right)^{\frac{1}{\tau}}}\left(\int_{Q}|f|^{\tau} d \mu\right)^{\frac{1}{\tau}} \\
& \leq \sup _{Q_{0} \subset Q \in \mathscr{Q}(\mu)} \mu\left(\frac{2 \eta}{1+\eta} Q\right)^{\frac{1}{p}-\frac{1}{q}}\left(\int_{Q}|f|^{q} d \mu\right)^{\frac{1}{q}}
\end{aligned}
$$

which implies the lemma.

Lemma 3.2. Let $1<\tau<\frac{n}{\alpha}, 1<\tau<q \leq p<\infty, 1<t \leq s<\infty, \frac{1}{s}=\frac{1}{p}-\frac{\alpha}{n}$, $\frac{q}{p}=\frac{t}{s}$, then

$$
\left\|M_{\tau, \eta}^{(\alpha)} f\right\|_{M_{t}^{s}(\mu)} \leq C\|f\|_{M_{q}^{p}(\mu)}
$$

with the constant $C$ independent of $f$.

Proof. Noting the condition $\frac{q}{p}=\frac{t}{s}$, it is enough to show the following pointwise estimates

$$
M_{\tau, \eta}^{(\alpha)}(f)(x) \leq C\left(\|f\|_{M_{q}^{p}(\mu)}\right)^{1-\frac{p}{s}}\left(M_{\tau, \eta} f(x)\right)^{\frac{p}{s}} .
$$

To do this, we fixed the point $x$ such that $M_{\tau, \eta}^{(\alpha)}(f)(x)>0$. We choose $\beta_{x}=$ $\left(\frac{\|f\|_{M_{q}^{p}(\mu)}}{M_{\tau, \eta} f(x)}\right)^{p}$ and then write

$$
\begin{aligned}
M_{\tau, \eta}^{(\alpha)} f(x) \leq & \sup _{\substack{x \in Q \in \mathcal{Q}(\mu) \\
\mu(\eta Q) \leq \beta_{x}}}\left(\frac{1}{\mu(\eta Q)^{1-\frac{\tau \alpha}{n}}} \int_{Q}|f(y)|^{\tau} d \mu(y)\right)^{\frac{1}{\tau}} \\
& +\sup _{\substack{x \in Q \in \mathcal{Q}(\mu) \\
\mu(\eta Q)>\beta_{x}}}\left(\frac{1}{\mu(\eta Q)^{1-\frac{\tau \alpha}{n}}} \int_{Q}|f(y)|^{\tau} d \mu(y)\right)^{\frac{1}{\tau}} \\
\leq & \left(\beta_{x}\right)^{\frac{\alpha}{n}} \sup _{\substack{x \in Q \in \mathcal{Q}(\mu) \\
\mu(\eta) \leq \beta_{x}}}\left(\frac{1}{\mu(\eta Q)} \int_{Q}|f(y)|^{\tau} d \mu(y)\right)^{\frac{1}{\tau}}
\end{aligned}
$$




$$
+\sup _{\substack{x \in Q \in \mathcal{Q}(\mu) \\ \mu(\eta Q)>\beta x}} \mu(\eta Q)^{-\left(\frac{1}{p}-\frac{\alpha}{n}\right)}\|f\|_{M_{q}^{p}(\mu)}
$$

by using the Hölder inequality. Hence we have

$$
M_{\tau, \eta}^{(\alpha)} f(x) \leq\left(\beta_{x}\right)^{\frac{\alpha}{n}} M_{\tau, \eta} f(x)+\left(\beta_{x}\right)^{-\frac{1}{s}}\|f\|_{M_{q}^{p}(\mu)},
$$

which yields the desired inequality (3.6). The lemma is proved.

Lemma 3.3. [5] Suppose that $1<q \leq p<\infty$, and there exists an increasing sequence of concentric doubling cubes, $I_{0} \subset I_{1} \subset \cdots \subset I_{k} \subset \cdots$, such that

$$
\lim _{k \rightarrow \infty} m_{I_{k}}(f)=0, \quad \text { and } \quad \bigcup_{k=0}^{\infty} I_{k}=\mathbb{R}^{d} .
$$

Then there exist a constant $C>0$ independent on $f$ such that

$$
\|N f\|_{M_{q}^{p}(\mu)} \leq C\left\|M^{\sharp,(\alpha)} f\right\|_{M_{q}^{p}(\mu)} .
$$

In order to investigate the multilinear commutators $\left[\vec{b}, I_{\alpha, m}\right]$ and $\left[\vec{b}_{\sigma}, I_{\alpha, m}\right]$, we introduce the following decomposition technique. For any $\vec{\lambda}=\left(\lambda_{1}, \lambda_{2}, \ldots\right.$, $\left.\lambda_{m}\right) \in \mathbb{R}^{m}$, writing $\overrightarrow{b_{\sigma}}(x)-\overrightarrow{b_{\sigma}}(y)=\left(\overrightarrow{b_{\sigma}}(x)-\overrightarrow{\lambda_{\sigma}}\right)-\left(\overrightarrow{b_{\sigma}}(y)-\overrightarrow{\lambda_{\sigma}}\right)$, thus it is clear that $\left[\overrightarrow{b_{\sigma}}, I_{\alpha, m}\right](\vec{f})=\left[\left(\overrightarrow{b_{\sigma}}-\overrightarrow{\lambda_{\sigma}}\right), I_{\alpha, m}\right](\vec{f})$. Moreover,

$$
\begin{aligned}
{\left[\vec{b}, I_{\alpha, m}\right](\vec{f})(x)=} & \sum_{i=0}^{m} \sum_{\sigma \in C_{i}^{m}}(-1)^{m-i} \prod_{\sigma_{j} \in \sigma}\left(b_{\sigma_{j}}(x)-\lambda_{\sigma_{j}}\right) \\
& \times \int_{\left(\mathbb{R}^{d}\right)^{m}} \prod_{\sigma_{k} \in \sigma^{\prime}}\left(b_{\sigma_{k}}(y)-\lambda_{\sigma_{k}}\right) \\
& \times \frac{f_{1}\left(y_{1}\right) \cdots f_{m}\left(y_{m}\right) d \mu\left(y_{1}\right) \cdots d \mu\left(y_{m}\right)}{\left|\left(x-y_{1}, x-y_{2}, \ldots, x-y_{m}\right)\right|^{m n-\alpha}} .
\end{aligned}
$$

By expanding $b_{\sigma_{k}}(y)-\lambda_{\sigma_{k}}=\left(b_{\sigma_{k}}(y)-b_{\sigma_{k}}(x)\right)+\left(b_{\sigma_{k}}(x)-\lambda_{\sigma_{k}}\right)$ and

$$
\prod_{\sigma_{k} \in \sigma^{\prime}}\left(b_{\sigma_{k}}(y)-\lambda_{\sigma_{k}}\right)=\sum_{\substack{\sigma^{(1)} \cup \sigma^{(2)}=\sigma^{\prime} \\ \sigma^{(1)} \cap \sigma^{(2)}=\emptyset}} \prod_{\sigma_{u} \in \sigma^{(1)}}\left(b_{\sigma_{u}}(y)-b_{\sigma_{u}}(x)\right) \prod_{\sigma_{v} \in \sigma^{(2)}}\left(b_{\sigma_{v}}(x)-\lambda_{\sigma_{v}}\right),
$$

hence we can obtain from the equality (3.7) that

$$
\begin{aligned}
{\left[\vec{b}, I_{\alpha, m}\right](\vec{f})(x)=(} & -1)^{m+1} \prod_{j=1}^{m}\left(b_{j}(x)-\lambda_{j}\right) I_{\alpha, m}(\vec{f})(x) \\
& +(-1)^{m} I_{\alpha, m}((\vec{b}-\vec{\lambda}) \vec{f})(x) \\
& +\sum_{i=1}^{m-1} \sum_{\sigma \in C_{i}^{m}} C_{m, i} \prod_{\sigma_{j} \in \sigma}\left(b_{\sigma_{j}}(x)-\lambda_{\sigma_{j}}\right) \\
& \times\left[\overrightarrow{b_{\sigma^{\prime}}}, I_{\alpha, m}\right](\vec{f})(x),
\end{aligned}
$$

where $C_{m, i}$ are constants depending only on $m$ and $i$. 
Remember these notations and the equality (3.8) in mind, we turn to prove the following lemma, which is crucial to us.

Lemma 3.4. Let $0<\alpha<m n, \tau>1, s_{i}>1, b_{i} \in R B M O(\mu)$ and $f_{i} \in L^{q_{i}}(\mu)$, $i=1,2, \ldots, m$, then there exist a constant $C>0$ independent of $b_{i}$ and $f_{i}$ such that

$$
\begin{aligned}
M^{\sharp,(\alpha)}\left(\left[\vec{b}, I_{\alpha, m}\right](\vec{f})\right)(x) \leq & C\left[\left\|b_{1}\right\|_{*} \ldots\left\|b_{m}\right\|_{*} M_{\tau, \frac{3}{2}}\left(I_{\alpha, m}(\vec{f})\right)(x)\right. \\
& +\sum_{i=1}^{m-1} \sum_{\sigma \in C_{i}^{m}} \prod_{j \in \sigma}\left\|b_{j}\right\|_{*} M_{\tau, \frac{3}{2}} \\
& \times\left(\left[\overrightarrow{b_{\sigma^{\prime}}}, I_{\alpha, m}\right](\vec{f})\right)(x) \\
& \left.+\prod_{i=1}^{m}\left\|b_{i}\right\|_{*} M_{s_{i}, \frac{9}{8}}^{\left(\frac{\alpha}{m}\right)} f_{i}(x)\right] .
\end{aligned}
$$

Proof. For simplicity, we denote by $\mathscr{R}(\vec{b}, \vec{f})(x)$ the quantities on the right hand side of the inequality (3.9). Recall the definition of the sharp maximal operator $M^{\sharp,(\alpha)}$, and use the standard technique, see [3] for example, we only need to prove that, for any $x \in \mathbb{R}^{d}$ and a cube $Q \ni x$, there is a quantity $h_{Q}$ such that

$$
\frac{1}{\mu\left(\frac{3}{2} Q\right)} \int_{Q}\left|\left[\vec{b}, I_{\alpha, m}\right](\vec{f})(z)-h_{Q}\right| d \mu(z) \leq C \mathscr{R}(\vec{b}, \vec{f})(x)
$$

and

$$
\left|h_{Q}-h_{R}\right| \leq C\left(K_{Q, R} K_{Q, R}^{(\alpha)}\right)^{m} \mathscr{R}(\vec{b}, \vec{f})(x)
$$

with the absolute constant $C$ independent of $\vec{b}, \vec{f}, Q$ and $R$, where $R$ is any doubling cube with $Q \subset R$. In fact, we take

$$
\begin{aligned}
h_{Q}= & (-1)^{m} m_{Q}\left(I _ { \alpha , m } \left(\left(m_{\widetilde{Q}}\left(b_{1}\right)-b_{1}\right) f_{1} \chi_{\mathbb{R}^{d} \backslash \frac{4}{3} Q}, \ldots,\left(m_{\widetilde{Q}}\left(b_{m}\right)\right.\right.\right. \\
& \left.\left.\left.-b_{m}\right) f_{m} \chi_{\mathbb{R}^{d} \backslash \frac{4}{3} Q}\right)\right)
\end{aligned}
$$

and clearly

$$
\begin{aligned}
h_{R}= & (-1)^{m} m_{R}\left(I _ { \alpha , m } \left(\left(m_{R}\left(b_{1}\right)-b_{1}\right) f_{1} \chi_{\mathbb{R}^{d} \backslash \frac{4}{3} R}, \ldots,\left(m_{R}\left(b_{m}\right)\right.\right.\right. \\
& \left.\left.\left.-b_{m}\right) f_{m} \chi_{\mathbb{R}^{d} \backslash \frac{4}{3} R}\right)\right) .
\end{aligned}
$$


Recall the equality (3.8), for any $z \in Q$, we have that

$$
\begin{aligned}
\left|\left[\vec{b}, I_{\alpha, m}\right](\vec{f})(z)-h_{Q}\right| \leq & \left|\prod_{j=1}^{m}\left(b_{j}(x)-m_{\widetilde{Q}}\left(b_{j}\right)\right) I_{\alpha, m}(\vec{f})(z)\right| \\
& +\mid \sum_{i=1}^{m-1} \sum_{\sigma \in C_{i}^{m}} C_{m, i} \prod_{\sigma_{j} \in \sigma}\left(b_{\sigma_{j}}(z)-m_{\widetilde{Q}}\left(b_{\sigma_{j}}\right)\right) \\
& \times\left[\overrightarrow{b_{\sigma^{\prime}}}, I_{\alpha, m}\right](\vec{f})(z) \mid \\
& +\left|(-1)^{m} I_{\alpha, m}\left(\left(\vec{b}-m_{\widetilde{Q}}(\vec{b})\right) \vec{f}\right)(z)-h_{Q}\right| \\
=: & I(z)+I I(z)+I I I(z) .
\end{aligned}
$$

In order to show the inequality (3.10), we will calculate the integrals for the three functions above, respectively. Firstly, for $\tau>1$, by the Hölder inequality one sees that

$$
\begin{aligned}
& \frac{1}{\mu\left(\frac{3}{2} Q\right)} \int_{Q}|I(z)| d \mu(z) \\
& \leq \prod_{i=1}^{m}\left(\frac{1}{\mu\left(\frac{3}{2} Q\right)} \int_{Q}\left|\left(b_{i}(z)-m_{\widetilde{Q}}\left(b_{i}\right)\right)\right|^{\tau_{i}} d \mu(z)\right)^{\frac{1}{\tau_{i}}} \\
& \quad \times\left(\frac{1}{\mu\left(\frac{3}{2} Q\right)} \int_{Q}\left|I_{\alpha, m}(\vec{f})\right|^{\tau} d \mu(z)\right)^{\frac{1}{\tau}} \\
& \leq C\left\|b_{1}\right\|_{*}\left\|b_{2}\right\|_{*} \ldots\left\|b_{m}\right\|_{*} M_{\tau, \frac{3}{2}}\left(I_{\alpha, m}(\vec{f})\right)(x)
\end{aligned}
$$

where we have choose $\tau_{i}>1$ such that $\frac{1}{\tau_{1}}+\frac{1}{\tau_{2}}+\cdots+\frac{1}{\tau_{m}}+\frac{1}{\tau}=1$.

Similarly, for $\tau>1$, by the Hölder inequality, we also deduce that

$$
\begin{aligned}
& \frac{1}{\mu\left(\frac{3}{2} Q\right)} \int_{Q}|I I(z)| d \mu(z) \\
& \leq C \sum_{i=1}^{m-1} \sum_{\sigma \in C_{i}^{m}} \prod_{\sigma_{j} \in \sigma}\left\|b_{\sigma_{j}}\right\|_{*} M_{\tau, \frac{3}{2}}\left(\left[\overrightarrow{b_{\sigma^{\prime}}}, I_{\alpha, m}\right](\vec{f})\right)(x) .
\end{aligned}
$$

To estimate the integral related to the function $I I I(z)$, we split each $f_{i}$ as $f_{i}=f_{i}^{0}+f_{i}^{\infty}$, where $f_{i}^{0}=f \chi_{\frac{4}{3} Q}$ and $f_{i}^{\infty}=f_{i}-f_{i}^{0}$, this yields

$$
\begin{aligned}
\prod_{j=1}^{m} f_{j}\left(y_{j}\right) & =\sum_{\alpha_{1}, \alpha_{2}, \ldots, \alpha_{m} \in\{0, \infty\}} f_{1}^{\alpha_{1}}\left(y_{1}\right) \ldots f_{m}^{\alpha_{m}}\left(y_{m}\right) \\
& =\prod_{j=1}^{m} f_{j}^{0}\left(y_{j}\right)+\sum_{*} f_{1}^{\alpha_{1}}\left(y_{1}\right) \ldots f_{m}^{\alpha_{m}}\left(y_{m}\right)+\prod_{j=1}^{m} f_{j}^{\infty}\left(y_{j}\right),
\end{aligned}
$$

where each term in $\sum_{*}$ satisfies that $\alpha_{j_{1}}=\alpha_{j_{2}}=\cdots=\alpha_{j_{\lambda}}=0$, for some $1 \leq \lambda<m$ and some $\left\{j_{1}, j_{2}, \ldots, j_{\lambda}\right\} \subset\{1,2, \ldots, m\}$. So we can decompose 
the function $I I I(z)$ further into three parts as follows

$$
\begin{aligned}
I I I(z) \leq & \left|I_{\alpha, m}\left(\left(b_{1}-m_{\widetilde{Q}}\left(b_{1}\right)\right) f_{1}^{0}, \ldots,\left(b_{m}-m_{\widetilde{Q}}\left(b_{m}\right)\right) f_{m}^{0}\right)(z)\right| \\
& +\sum_{*}\left|I_{\alpha, m}\left(\left(b_{1}-m_{\widetilde{Q}}\left(b_{1}\right)\right) f_{1}^{\alpha_{1}}, \ldots,\left(b_{m}-m_{\widetilde{Q}}\left(b_{m}\right)\right) f_{m}^{\alpha_{m}}\right)(z)\right| \\
& +\mid(-1)^{m} I_{\alpha, m}\left(\left(b_{1}-m_{\widetilde{Q}}\left(b_{1}\right)\right) f_{1}^{\infty}, \ldots,\left(b_{m}\right.\right. \\
& \left.\left.-m_{\widetilde{Q}}\left(b_{m}\right)\right) f_{m}^{\infty}\right)(z)-h_{Q} \mid \\
=: & I I I_{1}(z)+I I I_{2}(z)+I I I_{3}(z) .
\end{aligned}
$$

For $s_{i}>1$, we can take $1<u_{i}<s_{i}$ such that $\frac{1}{v}=\frac{1}{u_{1}}+\frac{1}{u_{2}}+\cdots+$ $\frac{1}{u_{m}}-\frac{\alpha}{n}>0$ and $v>1$. Let $\frac{1}{u_{i}}=\frac{1}{s_{i}}+\frac{1}{t_{i}}$ for each $i=1,2, \ldots, m$, then $1<t_{i}<\infty$. Using Theorem 1.2, we know that the operator $I_{\alpha, m}$ is bounded from $L^{u_{1}}(\mu) \times L^{u_{2}}(\mu) \times \cdots \times L^{u_{m}}(\mu)$ to $L^{v}(\mu)$. Hence, by this boundedness and the Hölder inequality, we have

$$
\begin{aligned}
& \frac{1}{\mu\left(\frac{3}{2} Q\right)} \int_{Q}\left|I I I_{1}(z)\right| d \mu(z) \\
& \leq \frac{\mu(Q)^{1-\frac{1}{v}}}{\mu\left(\frac{3}{2} Q\right)}\left\|I_{\alpha, m}\left(\left(b_{1}-m_{\widetilde{Q}}\left(b_{1}\right)\right) f_{1}^{0}, \ldots,\left(b_{m}-m_{\widetilde{Q}}\left(b_{m}\right)\right) f_{m}^{0}\right)(z)\right\|_{L^{v}(\mu)} \\
& \leq C \mu\left(\frac{3}{2} Q\right)^{-\frac{1}{v}} \prod_{i=1}^{m}\left\|\left(b_{i}-m_{\widetilde{Q}}\left(b_{i}\right)\right) f_{i}^{0}\right\|_{L^{u_{i}}(\mu)} \\
& \leq C \mu\left(\frac{3}{2} Q\right)^{-\frac{1}{v}} \prod_{i=1}^{m}\left(\int_{\left(\frac{4}{3} Q\right)}\left|f_{i}\right|^{s_{i}} d \mu\left(y_{i}\right)\right)^{\frac{1}{s_{i}}} \\
& \quad \times\left(\int_{\left(\frac{4}{3} Q\right)}^{\left.\left|b_{i}-m_{\widetilde{Q}}\left(b_{i}\right)\right|^{t_{i}} d \mu\left(y_{i}\right)\right)^{\frac{1}{t_{i}}}}\right. \\
& \leq C \prod_{i=1}^{m}\left(\frac{1}{\mu\left(\frac{3}{2} Q\right)^{1-\frac{\alpha s_{i}}{m n}}} \int_{\left(\frac{4}{3} Q\right)}\left|f_{i}\right|^{s_{i}} d \mu\right)^{\frac{1}{s_{i}}} \\
& \quad \times\left(\frac{1}{\mu\left(\frac{3}{2} Q\right)} \int_{\left(\frac{4}{3} Q\right)}^{\left.\left|b_{i}-m_{\widetilde{Q}}\left(b_{i}\right)\right|^{t_{i}} d \mu\right)^{\frac{1}{t_{i}}}}\right. \\
& \leq C \prod_{i=1}^{m}\left\|b_{i}\right\|_{*} M_{s_{i}, \frac{9}{8}}^{\left(\frac{\alpha}{m}\right)} f_{i}(x) . \\
& \quad
\end{aligned}
$$

In order to estimate the integral of terms $I I I_{2}(z)$ and $I I I_{3}(z)$ over $Q$, we will give their point-wise estimates. In fact, for $z \in Q$, since $1 \leq \lambda \leq m-1$ we observe that 


$$
\begin{aligned}
& I I I_{2}(z)=\sum_{*}\left|I_{\alpha, m}\left(\left(b_{1}-m_{\widetilde{Q}}\left(b_{1}\right)\right) f_{1}^{\alpha_{1}}, \ldots,\left(b_{m}-m_{\widetilde{Q}}\left(b_{m}\right)\right) f_{m}^{\alpha_{m}}\right)(z)\right| \\
& \leq C \sum_{*} \prod_{j \in\left\{j_{1}, \ldots, j_{\lambda}\right\}} \int_{\frac{4}{3} Q}\left|b_{j}\left(y_{j}\right)-m_{\widetilde{Q}}\left(b_{j}\right)\right|\left|f_{j}\left(y_{j}\right)\right| d \mu\left(y_{j}\right) \\
& \times \int_{\left(\mathbb{R}^{d} \backslash \frac{4}{3} Q\right)^{m-\lambda}} \frac{\prod_{j \notin\left\{j_{1}, \ldots, j_{\lambda}\right\}}\left|b_{j}\left(y_{j}\right)-m_{\widetilde{Q}}\left(b_{j}\right)\right|\left|f_{j}\left(y_{j}\right)\right| d \mu\left(y_{j}\right)}{\left(\sum_{j \notin\left\{j_{1}, \ldots, j_{\lambda}\right\}}\left|z-y_{j}\right|\right)^{m n-\alpha}} \\
& \leq C \sum_{*} \mu\left(\frac{3}{2} Q\right)^{\lambda-\frac{\alpha \lambda}{m n}} \prod_{j \in\left\{j_{1}, \ldots, j_{\lambda}\right\}} \\
& \times\left(\frac{1}{\mu\left(\frac{3}{2} Q\right)^{1-\frac{\alpha s_{j}}{m n}}} \int_{\frac{4}{3} Q}\left|f_{j}\left(y_{j}\right)\right|^{s_{j}} d \mu\left(y_{j}\right)\right)^{\frac{1}{s_{j}}} \\
& \times\left(\frac{1}{\mu\left(\frac{3}{2} Q\right)} \int_{\frac{4}{3} Q}\left|b_{j}\left(y_{j}\right)-m_{\widetilde{Q}}\left(b_{j}\right)\right|^{s_{j}^{\prime}} d \mu\left(y_{j}\right)\right)^{\frac{1}{s_{j}^{\prime}}} \\
& \times \sum_{k=1}^{\infty} \int_{\left(2^{\left.k \frac{4}{3} Q\right)^{m-\lambda}}\right.} \frac{\prod_{j \notin\left\{j_{1}, \ldots, j_{\lambda}\right\}}\left|b_{j}\left(y_{j}\right)-m_{\widetilde{Q}}\left(b_{j}\right)\right|\left|f_{j}\left(y_{j}\right)\right| d \mu\left(y_{j}\right)}{l\left(2^{k} \frac{4}{3} Q\right)^{m n-\alpha}} \\
& \leq C \sum_{*}\left(\prod_{j \in\left\{j_{1}, \ldots, j_{\lambda}\right\}}\left\|b_{j}\right\|_{*} M_{s_{j}, \frac{9}{8}}^{\left(\frac{\alpha}{m}\right)} f_{j}(x)\right) \sum_{k=1}^{\infty} 2^{-(k-1)\left(n-\frac{\alpha}{m}\right) \lambda} \\
& \times \int_{\left(2^{k} \frac{4}{3} Q\right)^{m-\lambda}} \frac{\prod_{j \notin\left\{j_{1}, \ldots, j_{\lambda}\right\}}\left|b_{j}\left(y_{j}\right)-m_{\widetilde{Q}}\left(b_{j}\right)\right|\left|f_{j}\left(y_{j}\right)\right| d \mu\left(y_{j}\right)}{l\left(2^{k} \frac{4}{3} Q\right)^{\left(n-\frac{\alpha}{m}\right)(m-\lambda)}} \\
& \leq C \sum_{*}\left(\prod_{j \in\left\{j_{1}, \ldots, j_{\lambda}\right\}}\left\|b_{j}\right\|_{*} M_{s_{j}, \frac{9}{8}}^{\left(\frac{\alpha}{m}\right)} f_{j}(x)\right) \sum_{k=1}^{\infty} 2^{-(k-1)\left(n-\frac{\alpha}{m}\right) \lambda} \\
& \times \prod_{j \notin\left\{j_{1}, \ldots, j_{\lambda}\right\}} \frac{1}{l\left(2^{k} \frac{4}{3} Q\right)^{\left(n-\frac{\alpha}{m}\right)}} \int_{\left(2^{k} \frac{4}{3} Q\right)}\left|b_{j}\left(y_{j}\right)-m_{\widetilde{Q}}\left(b_{j}\right)\right|\left|f_{j}\left(y_{j}\right)\right| d \mu\left(y_{j}\right) \\
& \leq C \sum_{*}\left(\prod_{j \in\left\{j_{1}, \ldots, j_{\lambda}\right\}}\left\|b_{j}\right\|_{*} M_{s_{j}, \frac{9}{8}}^{\left(\frac{\alpha}{m}\right)} f_{j}(x)\right) \sum_{k=1}^{\infty} 2^{-(k-1)\left(n-\frac{\alpha}{m}\right) \lambda} \\
& \times \prod_{j \notin\left\{j_{1}, \ldots, j_{\lambda}\right\}}\left[\left(\frac{1}{l\left(2^{k} \frac{4}{3} Q\right)^{n}} \int_{\left(2^{k} \frac{4}{3} Q\right)}\left|b_{j}\left(y_{j}\right)-m_{\widetilde{Q}}\left(b_{j}\right)\right|^{s_{j}^{\prime}} d \mu\left(y_{j}\right)\right)^{\frac{1}{s_{j}^{\prime}}}\right. \\
& \left.\times\left(\frac{1}{l\left(2^{k} \frac{4}{3} Q\right)^{\left(n-\frac{\alpha s_{j}}{m}\right)}} \int_{\left(2^{k} \frac{4}{3} Q\right)}\left|f_{j}\left(y_{j}\right)\right|^{s_{j}} d \mu\left(y_{j}\right)\right)^{\frac{1}{s_{j}}}\right]
\end{aligned}
$$




$$
\begin{aligned}
\leq & C \sum_{*} \prod_{j \in\left\{j_{1}, \ldots, j_{\lambda}\right\}}\left\|b_{j}\right\|_{*} M_{s_{j}, \frac{9}{8}}^{\left(\frac{\alpha}{m}\right)} f_{j}(x) \sum_{k=1}^{\infty} 2^{-k\left(n-\frac{\alpha}{m}\right) \lambda} \\
& \times \prod_{j \notin\left\{j_{1}, \ldots, j_{\lambda}\right\}}\left\|b_{j}\right\|_{*}(k+1) M_{s_{j}, \frac{9}{8}}^{\left(\frac{\alpha}{m}\right)} f_{j}(x) \\
\leq & C \prod_{j=1}^{m}\left\|b_{j}\right\|_{*} M_{s_{j}, \frac{9}{8}}^{\left(\frac{\alpha}{m}\right)} f_{j}(x),
\end{aligned}
$$

where we have used the fact (see [2]) that, there is an absolute constant $C$ such that, for any $b \in R B M O$, integer $k \geq 0$ and cubes $Q$,

$$
\left|m \underset{2^{k \frac{4}{3} Q}}{\widetilde{2}}(b)-m_{\widetilde{Q}}(b)\right| \leq C\|b\|_{*} K_{\widetilde{Q}, 2^{k \frac{4}{3} Q}} \leq C\|b\|_{*} K_{Q, 2^{k} \frac{4}{3} Q} \leq C k\|b\|
$$

On the other hand, for $I I I_{3}(z)$, we note for any $z, y \in Q$ that

$$
\begin{aligned}
& \mid I_{\alpha, m}\left(\left(b_{1}-m_{\widetilde{Q}}\left(b_{1}\right)\right) f_{1}^{\infty}, \ldots,\left(b_{m}-m_{\widetilde{Q}}\left(b_{m}\right)\right) f_{m}^{\infty}\right)(z) \\
& -I_{\alpha, m}\left(\left(b_{1}-m_{\widetilde{Q}}\left(b_{1}\right)\right) f_{1}^{\infty}, \ldots,\left(b_{m}-m_{\widetilde{Q}}\left(b_{m}\right)\right) f_{m}^{\infty}\right)(y) \mid \\
& \leq \int_{\left(\mathbb{R}^{d} \backslash \frac{4}{3} Q\right)^{m}}\left|\frac{1}{\left|\left(z-y_{1}, \ldots, z-y_{m}\right)\right|^{m n-\alpha}}-\frac{1}{\left|\left(y-y_{1}, \ldots, y-y_{m}\right)\right|^{m n-\alpha}}\right| \\
& \times\left|\prod_{i=1}^{m}\left(b_{i}\left(y_{i}\right)-m_{\widetilde{Q}}\left(b_{i}\right)\right) f_{i}^{\infty}\right| d \mu\left(y_{1}\right) d \mu\left(y_{2}\right) \cdots d \mu\left(y_{m}\right) \\
& \leq C \int_{\left(\mathbb{R}^{d} \backslash \frac{4}{3} Q\right)^{m}} \frac{|z-y| \prod_{i=1}^{m}\left|\left(b_{i}\left(y_{i}\right)-m_{\widetilde{Q}}\left(b_{i}\right)\right) f_{i}^{\infty}\right|}{\left|\left(y-y_{1}, \ldots, y-y_{m}\right)\right|^{m n-\alpha+1}} \\
& \times d \mu\left(y_{1}\right) d \mu\left(y_{2}\right) \cdots d \mu\left(y_{m}\right) \\
& \leq C \prod_{i=1}^{m} \sum_{k=1}^{\infty} \int_{2^{k} \frac{4}{3} Q \backslash 2^{k-1} \frac{4}{3} Q} \frac{l(Q)^{\frac{1}{m}}}{l\left(2^{k} \frac{3}{2} Q\right)^{n-\frac{\alpha}{m}+\frac{1}{m}}}\left|\left(b_{i}\left(y_{i}\right)-m_{\widetilde{Q}}\left(b_{i}\right)\right) f_{i}\right| d \mu\left(y_{i}\right) \\
& \leq C \prod_{i=1}^{m} \sum_{k=1}^{\infty} 2^{-k / m}\left(\frac{1}{l\left(2^{k} \frac{3}{2} Q\right)^{n}} \int_{2^{k} \frac{4}{3} Q}\left|\left(b_{i}\left(y_{i}\right)-m_{\widetilde{Q}}\left(b_{i}\right)\right)\right|^{s_{i}{ }^{\prime}} d \mu\left(y_{i}\right)\right)^{\frac{1}{s_{i}{ }^{\prime}}} \\
& \times\left(\frac{1}{l\left(2^{k} \frac{3}{2} Q\right)^{n-\left(\alpha s_{i} / m\right)}} \int_{2^{k} \frac{4}{3} Q}\left|f_{i}\left(y_{i}\right)\right|^{s_{i}} d \mu\left(y_{i}\right)\right)^{\frac{1}{s_{i}}} \\
& \leq C \prod_{i=1}^{m} \sum_{k=1}^{\infty} 2^{-k / m}(k+1)\left\|b_{i}\right\|_{*} M_{s_{i}, \frac{9}{8}}^{\left(\frac{\alpha}{m}\right)} f_{i}(x) \leq C \prod_{i=1}^{m}\left\|b_{i}\right\|_{*} M_{s_{i}, \frac{9}{8}}^{\left(\frac{\alpha}{m}\right)} f_{i}(x)
\end{aligned}
$$

where we have used the fact (3.18) again.

Taking the mean over $y \in Q$, we can obtain that

$$
\frac{1}{\mu\left(\frac{3}{2} Q\right)} \int_{Q}\left(\left|I I I_{2}(z)\right|+\left|I I I_{3}(z)\right|\right) d \mu(z) \leq C \prod_{i=1}^{m}\left\|b_{i}\right\|_{*} M_{s_{i}, \frac{9}{8}}^{\left(\frac{\alpha}{m}\right)} f_{i}(x) .
$$


Combing the inequalities (3.12), (3.13), (3.14), (3.16), (3.17) and (3.19), we see from the estimates of $I, I I, I I I_{1}, I I I_{2}$ and $I I I_{3}$ that the desired inequality (3.10) holds.

Next we turn to estimate the inequality (3.11). For any cubes $Q \subset R$ with $x \in Q$, where $R$ is doubling. We denote $N_{Q, R}+2$ by $N$, then $2^{N} Q \supset 2 Q$ and $2^{N} Q \supset 2 R$. We recall the equality (3.15) and let $f_{i}^{0}=f_{i} \chi_{2^{N} Q \backslash \frac{4}{3} Q}$ and $f_{i}^{R}=f_{i} \chi_{2^{N} Q \backslash \frac{4}{3} R}$, and let $f_{i}^{\infty}=f_{i} \chi_{\mathbb{R}^{d} \backslash 2^{N} Q}$. Then we can write

$$
\begin{aligned}
& \left|h_{Q}-h_{R}\right| \\
& =\mid m_{Q}\left[I_{\alpha, m}\left(\left(m_{\widetilde{Q}}\left(b_{1}\right)-b_{1}\right) f_{1} \chi_{\mathbb{R}^{d} \backslash \frac{4}{3} Q}, \ldots,\left(m_{\widetilde{Q}}\left(b_{m}\right)-b_{m}\right) f_{m} \chi_{\mathbb{R}^{d} \backslash \frac{4}{3} Q}\right)\right] \\
& -m_{R}\left[I_{\alpha, m}\left(\left(m_{R}\left(b_{1}\right)-b_{1}\right) f_{1} \chi_{\mathbb{R}^{d} \backslash \frac{4}{3} R}, \ldots,\left(m_{R}\left(b_{m}\right)-b_{m}\right) f_{m} \chi_{\mathbb{R}^{d} \backslash \frac{4}{3} R}\right)\right] \mid \\
& \leq \mid m_{Q}\left[I_{\alpha, m}\left(\left(m_{\widetilde{Q}}\left(b_{1}\right)-b_{1}\right) f_{1}^{\infty}, \ldots,\left(m_{\widetilde{Q}}\left(b_{m}\right)-b_{m}\right) f_{m}^{\infty}\right)\right] \\
& -m_{R}\left[I_{\alpha, m}\left(\left(m_{\widetilde{Q}}\left(b_{1}\right)-b_{1}\right) f_{1}^{\infty}, \ldots,\left(m_{\widetilde{Q}}\left(b_{m}\right)-b_{m}\right) f_{m}^{\infty}\right)\right] \mid \\
& +\mid m_{R}\left[I_{\alpha, m}\left(\left(m_{\widetilde{Q}}\left(b_{1}\right)-b_{1}\right) f_{1}^{\infty}, \ldots,\left(m_{\widetilde{Q}}\left(b_{m}\right)-b_{m}\right) f_{m}^{\infty}\right)\right] \\
& -m_{R}\left[I_{\alpha, m}\left(\left(m_{R}\left(b_{1}\right)-b_{1}\right) f_{1}^{\infty}, \ldots,\left(m_{R}\left(b_{m}\right)-b_{m}\right) f_{m}^{\infty}\right)\right] \mid \\
& +\sum_{\alpha_{1}, \alpha_{2}, \ldots, \alpha_{m} \in\{0, \infty\}} \mid m_{Q}\left[I _ { \alpha , m } \left(\left(m_{\widetilde{Q}}\left(b_{1}\right)-b_{1}\right) f_{1}^{\alpha_{1}}, \ldots,\right.\right. \\
& \text { at least one } \alpha_{i} \neq \infty \\
& \left.\left.\times\left(m_{\widetilde{Q}}\left(b_{m}\right)-b_{m}\right) f_{m}^{\alpha_{m}}\right)\right] \mid \\
& +\sum_{\alpha_{1}, \alpha_{2}, \ldots, \alpha_{m} \in\{R, \infty\}} \mid m_{R}\left[I _ { \alpha , m } \left(\left(m_{R}\left(b_{1}\right)-b_{1}\right) f_{1}^{\alpha_{1}}, \ldots,\right.\right. \\
& \text { at least one } \alpha_{i} \neq \infty \\
& \left.\left.\times\left(m_{R}\left(b_{m}\right)-b_{m}\right) f_{m}^{\alpha_{m}}\right)\right] \mid \\
& =A_{1}+A_{2}+A_{3}+A_{4} \text {. }
\end{aligned}
$$

For the term $A_{1}$, noting

$$
\left|m_{R}\left(b_{i}\right)-m_{\widetilde{Q}}\left(b_{i}\right)\right| \leq C\left\|b_{i}\right\|_{*} K_{Q, R}
$$

and the similar argument as that for the estimate of $I I I_{3}$, we can obtain that

$$
A_{1} \leq C \prod_{i=1}^{m} K_{Q, R}\left\|b_{i}\right\|_{*} M_{s_{i}, \frac{9}{8}}^{\left(\frac{\alpha}{m}\right)} f_{i}(x) \leq C\left(K_{Q, R}\right)^{m} \prod_{i=1}^{m}\left\|b_{i}\right\|_{*} M_{s_{i}, \frac{9}{8}}^{\left(\frac{\alpha}{m}\right)} f_{i}(x) \text {. }
$$

To estimate $A_{2}$, we recall the notations and note that, for any sequences $\xi_{j}$ and $\zeta_{j}$,

$$
\prod_{j=1}^{m}\left(\xi_{j}+\zeta_{j}\right)=\sum_{i=0}^{m} \sum_{\sigma \in C_{i}^{m}} \prod_{j \in \sigma} \xi_{j} \prod_{j^{\prime} \in \sigma^{\prime}} \zeta_{j^{\prime}}
$$


Using this equality and expanding $m_{\widetilde{Q}}\left(b_{j}\right)-b_{j}(y)=\left(m_{\widetilde{Q}}\left(b_{j}\right)-b_{j}(z)\right)+\left(b_{j}(z)-\right.$ $\left.b_{j}(y)\right)$, we observe that

$$
\begin{aligned}
I_{\alpha, m} & \left(\left(m_{\widetilde{Q}}\left(b_{1}\right)-b_{1}\right) f_{1}^{\infty}, \ldots,\left(m_{\widetilde{Q}}\left(b_{m}\right)-b_{m}\right) f_{m}^{\infty}\right)(z) \\
= & \sum_{i=0}^{m} \sum_{\sigma \in C_{i}^{m}} \prod_{j \in \sigma}\left(m_{\widetilde{Q}}\left(b_{j}\right)-b_{j}(z)\right)\left[\vec{b}_{\sigma^{\prime}}, I_{\alpha, m}\right]\left(\vec{f} \chi_{\mathbb{R}^{d} \backslash 2^{N} Q}\right)(z) .
\end{aligned}
$$

Similarly,

$$
\begin{aligned}
& I_{\alpha, m}\left(\left(m_{R}\left(b_{1}\right)-b_{1}\right) f_{1}^{\infty}, \ldots,\left(m_{R}\left(b_{m}\right)-b_{m}\right) f_{m}^{\infty}\right)(z) \\
& \quad=\sum_{i=0}^{m} \sum_{\sigma \in C_{i}^{m}} \prod_{j \in \sigma}\left(m_{R}\left(b_{j}\right)-b_{j}(z)\right)\left[\vec{b}_{\sigma^{\prime}}, I_{\alpha, m}\right]\left(\vec{f} \chi_{\mathbb{R}^{d} \backslash 2^{N} Q}\right)(z) .
\end{aligned}
$$

Thus

$$
\begin{aligned}
\mid I_{\alpha, m} & \left(\left(m_{\widetilde{Q}}\left(b_{1}\right)-b_{1}\right) f_{1}^{\infty}, \ldots,\left(m_{\widetilde{Q}}\left(b_{m}\right)-b_{m}\right) f_{m}^{\infty}\right)(z) \\
& -I_{\alpha, m}\left(\left(m_{R}\left(b_{1}\right)-b_{1}\right) f_{1}^{\infty}, \ldots,\left(m_{R}\left(b_{m}\right)-b_{m}\right) f_{m}^{\infty}\right)(z) \mid \\
\leq & \left|\sum_{i=1}^{m} \sum_{\sigma \in C_{i}^{m}} \prod_{j \in \sigma}\left(m_{\widetilde{Q}}\left(b_{j}\right)-b_{j}(z)\right)\left[\vec{b}_{\sigma^{\prime}}, I_{\alpha, m}\right]\left(\vec{f} \chi_{\mathbb{R}^{d} \backslash 2^{N} Q}\right)(z)\right| \\
& +\left|\sum_{i=1}^{m} \sum_{\sigma \in C_{i}^{m}} \prod_{j \in \sigma}\left(m_{R}\left(b_{j}\right)-b_{j}(z)\right)\left[\vec{b}_{\sigma^{\prime}}, I_{\alpha, m}\right]\left(\vec{f} \chi_{\mathbb{R}^{d} \backslash 2^{N} Q}\right)(z)\right| \\
= & : B_{1}(z)+B_{2}(z) .
\end{aligned}
$$

To estimate the integrals above, we recall that $f_{j}^{\infty}=f_{j} \chi_{\mathbb{R}^{d} \backslash 2^{N} Q}$ and let $f_{j}^{Q}=f_{j} \chi_{2^{N} Q}$, then we can write that $f_{j}^{\infty}=f_{j}-f_{j}^{Q}$ and $f_{j}=f_{j}^{\infty}+f_{j}^{Q}$, and thus we have

$$
\begin{aligned}
\prod_{j=1}^{m} f_{j}^{\infty}\left(y_{j}\right)= & \prod_{j=1}^{m} f_{j}\left(y_{j}\right)+\sum_{i=1}^{m}(-1)^{i} \sum_{\varrho \in C_{i}^{m}} \prod_{j \in \varrho} f_{j}^{Q}\left(y_{j}\right) \prod_{j^{\prime} \in \varrho^{\prime}} f_{j^{\prime}}\left(y_{j^{\prime}}\right) \\
= & \prod_{j=1}^{m} f_{j}\left(y_{j}\right)+\sum_{\lambda=1}^{m} \sum_{\left\{j_{1}, j_{2}, \ldots, j_{\lambda}\right\} \subset\{1,2, \ldots, m\}} \\
& \times C_{j_{1}, j_{2}, \ldots, j_{m}} f_{j_{1}}^{Q}\left(y_{j_{1}}\right) \ldots f_{j_{\lambda}}^{Q}\left(y_{j_{\lambda}}\right) f_{j_{\lambda+1}}^{\infty}\left(y_{j_{\lambda+1}}\right) \ldots f_{j_{m}}^{\infty}\left(y_{j_{m}}\right)
\end{aligned}
$$

where $C_{j_{1}, j_{2}, \ldots, j_{m}}$ are constants independent of $\vec{f}$ and $Q$. From the equality (3.22), we can deduce that

$$
\begin{aligned}
& {\left[\vec{b}_{\sigma^{\prime}}, I_{\alpha, m}\right]\left(\vec{f} \chi_{\mathbb{R}^{d} \backslash 2^{N} Q}\right)(x)=\left[\vec{b}_{\sigma^{\prime}}, I_{\alpha, m}\right](\vec{f})(z)} \\
& \quad+\sum_{\lambda=1}^{m} \sum_{\left\{j_{1}, j_{2}, \ldots, j_{\lambda}\right\} \subset\{1,2, \ldots, m\}} C_{j_{1}, j_{2}, \ldots, j_{\lambda}} I_{\alpha, m}\left(g_{1} F_{1}, g_{2} F_{2}, \ldots, g_{m} F_{m}\right)(z)
\end{aligned}
$$


where

$$
g_{j}(y)= \begin{cases}b_{j}(z)-b_{j}(y), & \text { if } j \in \sigma^{\prime} \\ 1, & \text { if } j \in \sigma\end{cases}
$$

and

$$
F_{j}(y)=\left\{\begin{array}{ll}
f_{j}^{Q}(y), & \text { if } j \in\left\{j_{1}, j_{2}, \ldots, j_{\lambda}\right\} \\
f_{j}^{\infty}(y), & \text { if } j \notin\left\{j_{1}, j_{2}, \ldots, j_{\lambda}\right\}
\end{array} .\right.
$$

Along the same lines as that of the pointwise estimates of $I I I_{2}(z)$, we can obtain that, for $x, z \in R \subset 2^{N-1} Q$ and if $1 \leq \lambda \leq m$,

$$
\begin{aligned}
& \left|I_{\alpha, m}\left(g_{1} F_{1}, g_{2} F_{2}, \ldots, g_{m} F_{m}\right)(z)\right| \\
& \leq C \prod_{j \in\left\{j_{1}, j_{2}, \ldots, j_{\lambda}\right\}} \frac{1}{l\left(2^{N} Q\right)^{n-\frac{\alpha}{m}}} \int_{2^{N} Q}\left|g_{j}\left(y_{j}\right) f_{j}\left(y_{j}\right)\right| d \mu\left(y_{j}\right) \\
& \quad \times \sum_{k=1}^{\infty} 2^{-k\left(n-\frac{\alpha}{m}\right) \lambda} \prod_{\substack{j \notin\left\{j_{1}, j_{1}, \ldots, j_{\lambda}\right\} \\
1 \leq j \leq m}} \frac{1}{l\left(2^{k} 2^{N} Q\right)^{n-\frac{\alpha}{m}}} \\
& \quad \times \int_{2^{k} 2^{N} Q}\left|g_{j}\left(y_{j}\right) f_{j}\left(y_{j}\right)\right| d \mu\left(y_{j}\right) .
\end{aligned}
$$

Let $\left(g_{j}\right)_{k, s_{j}^{\prime}}^{*}=1$ if $j \in \sigma ;$ and

$$
\left(g_{j}\right)_{k, s_{j}^{\prime}}^{*}=\left\{\begin{array}{cc}
\left(\frac{1}{l\left(2^{N} Q\right)^{n}} \int_{2^{N} Q}\left|b_{j}(z)-b_{j}(y)\right|^{s_{j}^{\prime}} d \mu(y)\right)^{\frac{1}{s_{j}^{\prime}},} & \text { if } j \in \sigma^{\prime} \cap \\
\left(\frac{1}{l\left(2^{k+N} Q\right)^{n}} \int_{2^{k+N} Q}\left|b_{j}(z)-b_{j}(y)\right|^{s_{j}^{\prime}} d \mu(y)\right)^{\frac{1}{s_{j}^{\prime}},} & \text { if } \left.j \in \sigma_{1}, j_{2}, \ldots, j_{\lambda}\right\} \\
& \left\{j_{1}, j_{2}, \ldots, j_{\lambda}\right\}
\end{array}\right.
$$

thus for $j \in \sigma^{\prime}$,

$$
\left(g_{j}\right)_{k, s_{j}^{\prime}}^{*} \leq C|| b_{j} \|_{*}+C\left|m_{2^{N} Q}\left(b_{j}\right)-b_{j}(z)\right|+C\left|m_{2^{k+N} Q}\left(b_{j}\right)-b_{j}(z)\right| .
$$

Hence we get from (3.24) that, for $\tau>1$,

$$
\begin{aligned}
& \left(\frac{1}{\mu(R)} \int_{R}\left|I_{\alpha, m}\left(g_{1} F_{1}, g_{2} F_{2}, \ldots, g_{m} F_{m}\right)(z)\right|^{\tau} d \mu(z)\right)^{\frac{1}{\tau}} \\
& \leq C\left(\prod_{j=1}^{m} M_{s_{j}, \frac{9}{8}}^{\left(\frac{\alpha}{m}\right)} f_{j}(x)\right) \sum_{k=1}^{\infty} 2^{-k\left(n-\frac{\alpha}{m}\right) \lambda} \\
& \quad \times\left(\frac{1}{\mu(R)} \int_{R}\left|\prod_{j \in \sigma^{\prime}}\left(g_{j}\right)_{k, s_{j}^{\prime}}^{*}\right|^{\tau} d \mu(z)\right)^{\frac{1}{\tau}}
\end{aligned}
$$




$$
\begin{aligned}
\leq & C\left(\prod_{j=1}^{m} M_{s_{j}, \frac{9}{8}}^{\left(\frac{\alpha}{m}\right)} f_{j}(x)\right) \sum_{k=1}^{\infty} 2^{-k\left(n-\frac{\alpha}{m}\right) \lambda} \prod_{j \in \sigma^{\prime}}\left\|b_{j}\right\|_{*} \\
& \times\left(1+K_{R, 2^{N} Q}+K_{R, 2^{k+N} Q}\right) \\
\leq & C\left(\prod_{j=1}^{m} M_{s_{j}, \frac{9}{8}}^{\left(\frac{\alpha}{m}\right)} f_{j}(x)\right) \prod_{j \in \sigma^{\prime}}\left\|b_{j}\right\|_{*}
\end{aligned}
$$

where we have used the fact that the cubes $R$ and $2^{N} Q$ are comparable, which implies $K_{R, 2^{N} Q} \leq C$ and $K_{R, 2^{k+N} Q} \leq C(1+k)$. Using the inequality (3.25) above and the identity (3.23), we obtain that, for $\tau>1$,

$$
\begin{aligned}
A_{2} \leq & \frac{1}{\mu(R)} \int_{R}\left(\left|B_{1}(z)\right|+\left|B_{2}(z)\right|\right) d \mu(z) \\
\leq & C \sum_{i=1}^{m} \sum_{\sigma \in C_{i}^{m}}\left(\prod_{j \in \sigma} K_{Q, R}\left\|b_{j}\right\|_{*}\right) \\
& \times\left(\frac{1}{\mu(R)} \int_{R}\left|\left[\vec{b}_{\sigma^{\prime}}, I_{\alpha, m}\right]\left(\vec{f} \chi_{\mathbb{R}^{d} \backslash 2^{N} Q}\right)(z)\right|^{\tau} d \mu(z)\right)^{\frac{1}{\tau}} \\
\leq & C \sum_{i=1}^{m} \sum_{\sigma \in C_{i}^{m}}\left(\prod_{j \in \sigma} K_{Q, R}\left\|b_{j}\right\|_{*}\right) M_{\tau, \frac{3}{2}}\left(\left[\vec{b}_{\sigma^{\prime}}, I_{\alpha, m}\right](\vec{f})\right)(x) \\
& +C \prod_{j=1}^{m} K_{Q, R}\left\|b_{j}\right\|_{*} M_{s_{j}, \frac{9}{8}}^{\left(\frac{\alpha}{m}\right)} f_{j}(x) .
\end{aligned}
$$

The estimates of $A_{3}$ and $A_{4}$ is very similar to the one used in the estimate of $A_{2}$. In fact, repeating the similar procedures used in (3.24) and (3.25) for $\tau=1$, and noting that $K_{Q, 2^{k+N} Q} \leq K_{Q, R}+K_{R, 2^{k+N} Q} \leq C(1+k)+K_{Q, R}$ since $2^{N-3} Q \subset 2 R \subset 2^{N} Q$ by the definition of $N$, we can deduce that

$$
\begin{aligned}
& \quad \sum_{\begin{array}{c}
\alpha_{1}, \alpha_{2}, \ldots, \alpha_{m} \in\{0, \infty\} \\
\text { at least one } \alpha_{i \neq \infty} \\
\text { and one } \alpha_{j} \neq 0
\end{array}}\left|m_{Q}\left[I_{\alpha, m}\left(\left(m_{\widetilde{Q}}\left(b_{1}\right)-b_{1}\right) f_{1}^{\alpha_{1}}, \ldots,\left(m_{\widetilde{Q}}\left(b_{m}\right)-b_{m}\right) f_{m}^{\alpha_{m}}\right)\right]\right| \\
& \quad+\sum_{\substack{\alpha_{1}, \alpha_{2}, \ldots, \alpha_{m} \in\{R, \infty\} \\
\text { at least one } \alpha_{i} \neq \infty}} \mid m_{R}\left[I _ { \alpha , m } \left(\left(m_{R}\left(b_{1}\right)-b_{1}\right) f_{1}^{\alpha_{1}}, \ldots,\right.\right. \\
& \left.\left.\quad \times\left(m_{R}\left(b_{m}\right)-b_{m}\right) f_{m}^{\alpha_{m}}\right)\right] \mid \\
& \leq C \prod_{j=1}^{m} K_{Q, R}\left\|b_{j}\right\|_{*} M_{s_{j}, \frac{9}{8}}^{\left(\frac{\alpha}{m}\right)} f_{j}(x) .
\end{aligned}
$$

It is left to estimate the term in $A_{3}$ of the case $\alpha_{1}=\alpha_{2}=\cdots=\alpha_{m}=0$. A small modification is needed to estimate this term. For $z \in Q$ and $x \in Q$, one sees

$$
I_{\alpha, m}\left(\left(m_{\widetilde{Q}}\left(b_{1}\right)-b_{1}\right) f_{1}^{0}, \ldots,\left(m_{\widetilde{Q}}\left(b_{j}\right)-b_{j}\right) f_{j}^{0}, \ldots,\left(m_{\widetilde{Q}}\left(b_{m}\right)-b_{m}\right) f_{m}^{0}\right)
$$




$$
\begin{aligned}
\leq & C \sum_{k=1}^{N} \prod_{j=1}^{m} \frac{1}{l\left(2^{k} \frac{4}{3} Q\right)^{n-\frac{\alpha}{m}}} \int_{2^{k} \frac{4}{3} Q}\left|\left(m_{\widetilde{Q}}\left(b_{j}\right)-b_{j}\left(y_{j}\right)\right) f_{j}\left(y_{j}\right)\right| d \mu\left(y_{j}\right) \\
\leq & C \sum_{k=1}^{N} \prod_{j=1}^{m} \frac{1}{l\left(2^{k} \frac{4}{3} Q\right)^{n-\frac{\alpha}{m}}}\left(\int_{2^{k} \frac{4}{3} Q}\left|\left(m_{\widetilde{Q}}\left(b_{j}\right)-b_{j}\right)\right|^{s_{j}^{\prime}} d \mu\left(y_{j}\right)\right)^{\frac{1}{s_{j}^{\prime}}} \\
& \times\left(\int_{2^{k} \frac{4}{3} Q}\left|f_{j}\right|^{s_{j}} d \mu\left(y_{j}\right)\right)^{\frac{1}{s_{j}}} \\
\leq & C \sum_{k=1}^{N} \prod_{j=1}^{m} \frac{\mu\left(2^{k} \frac{3}{2} Q\right)^{1-\frac{\alpha}{m n}}}{l\left(2^{k} \frac{4}{3} Q\right)^{n-\frac{\alpha}{m}}}\left(1+K_{Q, \widetilde{Q}}+K_{Q, 2^{k} \frac{4}{3} Q}\right)\left\|b_{j}\right\|_{*} M_{s_{j}, \frac{9}{8}}^{\left(\frac{\alpha}{m}\right)} f_{j}(x) \\
\leq & C\left(K_{Q, R} K_{Q, R}^{\alpha}\right)^{m} \prod_{j=1}^{m}\left\|b_{j}\right\|_{*} M_{s_{j}, \frac{9}{8}}^{\left(\frac{\alpha}{m}\right)} f_{j}(x) .
\end{aligned}
$$

This and the inequality (3.26) follows

$$
A_{3}+A_{4} \leq C\left(K_{Q, R} K_{Q, R}^{(\alpha)}\right)^{m} \prod_{j=1}^{m}\left\|b_{j}\right\|_{*} M_{s_{j}, \frac{9}{8}}^{\left(\frac{\alpha}{m}\right)} f_{j}(x) .
$$

Moreover, combing the estimates of $A_{1}, A_{2}, A_{3}$ and $A_{4}$, we obtain the desired inequality (3.11).

Finally, let us show how to acquire the inequality (3.9) from the two inequalities (3.10) and (3.11). Fix the point $x$ and let $Q$ be any cube such that $x \in Q$, notice $K_{Q, \widetilde{Q}} \leq C$ and $K_{Q, \widetilde{Q}}^{(\alpha)} \leq C$, hence we see from the inequalities (3.10) and (3.11) that

$$
\begin{aligned}
& \frac{1}{\mu\left(\frac{3}{2} Q\right)} \int_{Q}\left|\left[\vec{b}, I_{\alpha, m}\right](\vec{f})(z)-m_{\widetilde{Q}}\left(\left[\vec{b}, I_{\alpha, m}\right](\vec{f})\right)\right| d \mu(z) \\
& \leq \frac{1}{\mu\left(\frac{3}{2} Q\right)} \int_{Q}\left|\left[\vec{b}, I_{\alpha, m}\right](\vec{f})(z)-h_{Q}\right| d \mu(z)+\frac{1}{\mu\left(\frac{3}{2} Q\right)} \int_{Q}\left|h_{Q}-h_{\widetilde{Q}}\right| d \mu(z) \\
& \quad+\frac{1}{\mu\left(\frac{3}{2} Q\right)} \int_{Q}\left|m_{\widetilde{Q}}\left[\vec{b}, I_{\alpha, m}\right](\vec{f})-h_{\widetilde{Q}}\right| d \mu(z) \\
& \leq C \mathscr{R}(\vec{b}, \vec{f})(x) .
\end{aligned}
$$

On the other hand, for all doubling cubes $Q \subset R$ with $x \in Q$ such that $K_{Q, R} \leq K_{Q, R}^{(\alpha)} \leq P_{0}$, where $P_{0}$ is the constant in Lemma 6 in [3], using (3.11), we have

$$
\left|h_{Q}-h_{R}\right| \leq C K_{Q, R}^{(\alpha)} P_{0}^{2 m-1} \mathscr{R}(\vec{b}, \vec{f})(x),
$$


and moreover the inequality (3.28) holds for any doubling cubes $Q, R$ with $Q \subset R$. Therefore,

$$
\begin{aligned}
& \mid m_{Q}\left(\left[\vec{b}, I_{\alpha, m}\right](\vec{f})\right)-m_{R}\left(\left[\vec{b}, I_{\alpha, m}\right](\vec{f})\right) \mid \\
& \leq\left|m_{Q}\left(\left[\vec{b}, I_{\alpha, m}\right](\vec{f})\right)-h_{Q}\right|+\left|h_{R}-m_{R}\left(\left[\vec{b}, I_{\alpha, m}\right](\vec{f})\right)\right| \\
& \quad+\left|h_{Q}-h_{R}\right| \\
& \leq C K_{Q, R}^{(\alpha)} \mathscr{R}(\vec{b}, \vec{f})(x) .
\end{aligned}
$$

According to the estimates (3.27), (3.29) and the definition of the sharp maximal function, we deduce the inequality (3.9) and so finish the proof of the lemma.

Corollary 3.5. Using the same notations in Lemma 3.4, and letting $\sigma \subset$ $\{1,2, \ldots, m\}$, we have

$$
\begin{aligned}
& M^{\sharp,(\alpha)}\left(\left[\vec{b}_{\sigma}, I_{\alpha, m}\right](\vec{f})\right)(x) \leq C\left[\prod_{j \in \sigma}\left\|b_{j}\right\|_{*} M_{\tau, \frac{3}{2}}\left(I_{\alpha, m}(\vec{f})\right)(x)\right. \\
& +\sum_{\substack{\sigma, \cup \sigma_{2}=\sigma \\
\sigma_{1} \neq \emptyset, \sigma_{2} \neq \emptyset}} \prod_{j \in \sigma_{1}}\left\|b_{j}\right\|_{*} M_{\tau, \frac{3}{2}}\left(\left[\overrightarrow{b_{\sigma_{2}}}, I_{\alpha, m}\right](\vec{f})\right)(x) \\
& \left.+\prod_{j \in \sigma}\left\|b_{j}\right\|_{*} \prod_{j=1}^{m} M_{s_{j}, \frac{9}{8}}^{\left(\frac{\alpha}{m}\right)} f_{j}(x)\right]
\end{aligned}
$$

and particular,

$$
M^{\sharp,(\alpha)}\left(\left[b_{i}, I_{\alpha, m}\right](\vec{f})\right)(x) \leq C\left\|b_{i}\right\|_{*}\left[M_{\tau, \frac{3}{2}}\left(I_{\alpha, m}(\vec{f})\right)(x)+\prod_{j=1}^{m} M_{s_{j}, \frac{9}{8}}^{\left(\frac{\alpha}{m}\right)} f_{j}(x)\right] .
$$

Proof. The proof of (3.30) and (3.31) is very similar to (3.9), so we omit the details.

Now we are ready to give the proof of theorem 1.4. Using Lemma 3.2, Lemma 3.3, Corollary 3.5 and Theorem 1.2, we get that

$$
\begin{aligned}
\left\|\left[b_{i}, I_{\alpha, m}\right](\vec{f})\right\|_{M_{q}^{p}(\mu)} & \leq C\left\|N\left(\left[b_{i}, I_{\alpha, m}\right](\vec{f})\right)\right\|_{M_{q}^{p}(\mu)} \\
& \leq C\left\|M^{\sharp,(\alpha)}\left(\left[b_{i}, I_{\alpha, m}\right](\vec{f})\right)\right\|_{M_{q}^{p}(\mu)} \\
& \leq C\left\|b_{i}\right\|_{*}\left\|M_{\tau, \frac{3}{2}}\left(I_{\alpha, m}(\vec{f})\right)(x)+\prod_{i=1}^{m} M_{s_{i}, \frac{9}{8}}^{\left(\frac{\alpha}{m}\right)} f_{i}\right\|_{M_{q}^{p}(\mu)} \\
& \leq C\left\|b_{i}\right\|_{*}\left\|M_{\tau, \frac{3}{2}}\left(I_{\alpha, m}(\vec{f})\right)\right\|_{M_{q}^{p}(\mu)}+C\left\|b_{i}\right\|_{*}
\end{aligned}
$$


Vol. 18 (2011)

$$
\begin{aligned}
& \times\left\|\prod_{i=1}^{m} M_{s_{i}, \frac{9}{8}}^{\left(\frac{\alpha}{m}\right)} f_{i}\right\|_{M_{q}^{p}(\mu)} \\
\leq & C\left\|b_{i}\right\|_{*}\left\|I_{\alpha, m}(\vec{f})\right\|_{M_{q}^{p}(\mu)}+C\left\|b_{i}\right\|_{*} \prod_{i=1}^{m}\left\|f_{i}\right\|_{M_{q_{i}}^{p_{i}}(\mu)} \\
\leq & C\left\|b_{i}\right\|_{*} \prod_{j=1}^{m}\left\|f_{j}\right\|_{M_{q_{j}}^{p_{j}}(\mu)} .
\end{aligned}
$$

Similarly, applying the inequality (3.30) in Corollary 3.5, for $\sigma \subseteq\{1,2, \ldots, m\}$, we have

$$
\begin{aligned}
& \left\|\left[\vec{b}_{\sigma}, I_{\alpha, m}\right](\vec{f})\right\|_{M_{q}^{p}(\mu)} \leq C\left\|N\left(\left[\vec{b}_{\sigma}, I_{\alpha, m}\right](\vec{f})\right)\right\|_{M_{q}^{p}(\mu)} \\
& \leq C\left\|M^{\sharp,(\alpha)}\left[\vec{b}_{\sigma}, I_{\alpha, m}\right](\vec{f})\right\|_{M_{q}^{p}(\mu)} \\
& \leq C\left\|\prod_{j \in \sigma}\right\| b_{j} \|_{*} M_{\tau, \frac{3}{2}}\left(I_{\alpha, m}(\vec{f})\right) \\
& +\sum_{\substack{\sigma_{1} \cup \sigma_{2}=\sigma \\
\sigma_{1} \neq \emptyset, \sigma_{2} \neq \emptyset}} \prod_{j \in \sigma_{1}}\left\|b_{j}\right\|_{*} M_{\tau, \frac{3}{2}}\left(\left[\overrightarrow{b_{\sigma_{2}}}, I_{\alpha, m}\right](\vec{f})\right) \\
& +\prod_{j \in \sigma}\left\|b_{j}\right\|_{*} \prod_{j=1}^{m} M_{s_{j}, \frac{9}{8}}^{\left(\frac{\alpha}{m}\right)} f_{j} \|_{M_{q}^{p}(\mu)} \\
& \leq C \prod_{j \in \sigma}\left\|b_{j}\right\|_{*}\left\|M_{\tau, \frac{3}{2}}\left(I_{\alpha, m}(\vec{f})\right)\right\|_{M_{q}^{p}(\mu)} \\
& +C \sum_{\substack{\sigma_{1} \cup \sigma_{2}=\sigma \\
\sigma_{1} \neq \emptyset, \sigma_{2} \neq \emptyset}} \prod_{j \in \sigma_{1}}\left\|b_{j}\right\|_{*}\left\|M_{\tau, \frac{3}{2}}\left(\left[\overrightarrow{b_{\sigma_{2}}}, I_{\alpha, m}\right](\vec{f})\right)\right\|_{M_{q}^{p}(\mu)} \\
& +C \prod_{j \in \sigma}\left\|b_{j}\right\|_{*}\left\|\prod_{j=1}^{m} M_{s_{j}, \frac{9}{8}}^{\left(\frac{\alpha}{m}\right)} f_{j}\right\|_{M_{q}^{p}(\mu)} \\
& \leq C \prod_{j \in \sigma}\left\|b_{j}\right\|_{*} \prod_{j=1}^{m}\left\|f_{j}\right\|_{M_{q_{j}}^{p_{j}}(\mu)} \\
& +C \sum_{\substack{\sigma_{1} \cup \sigma_{2}=\sigma \\
\sigma_{1} \neq \emptyset, \sigma_{2} \neq \emptyset}} \prod_{j \in \sigma_{1}}\left\|b_{j}\right\|_{*}\left\|M_{\tau, \frac{3}{2}}\left(\left[\overrightarrow{b_{\sigma_{2}}}, I_{\alpha, m}\right](\vec{f})\right)\right\|_{M_{q}^{p}(\mu)} \\
& \leq C \prod_{j \in \sigma}\left\|b_{j}\right\|_{*} \prod_{j=1}^{m}\left\|f_{j}\right\|_{M_{q_{j}}^{p_{j}}(\mu)} \\
& +C \sum_{\substack{\sigma_{1} \cup \sigma_{2}=\sigma \\
\sigma_{1} \neq \emptyset, \sigma_{2} \neq \emptyset}} \prod_{j \in \sigma_{1}}\left\|b_{j}\right\|_{*}\left\|\left[\overrightarrow{b_{\sigma_{2}}}, I_{\alpha, m}\right](\vec{f})\right\|_{M_{q}^{p}(\mu)}
\end{aligned}
$$


where $\sigma_{1}$ and $\sigma_{2}$ are two nonempty subsets of $\sigma$ and $\sigma_{1} \cap \sigma_{2}=\emptyset$. Hence, we can make use of induction on $\sigma \subseteq\{1,2, \ldots, m\}$ to get that

$$
\left\|\left[\vec{b}_{\sigma}, I_{\alpha, m}\right](\vec{f})\right\|_{M_{q}^{p}(\mu)} \leq C \prod_{j \in \sigma}\left\|b_{j}\right\|_{*} \prod_{i=1}^{m}\left\|f_{i}\right\|_{M_{q_{i}}^{p_{i}}(\mu)} .
$$

This completes the proof of Theorem 1.4.

\section{Acknowledgments}

The authors thank the anonymous referee for reading the paper carefully and giving several useful suggestions.

\section{References}

[1] Coifman, R., Rochberg, R., Weiss, G.: Factoriztion theorems for Hardy spaces in several variables. Ann. Math. 103, 611-635 (1976)

[2] Tolsa, X.: BMO, $H^{1}$ and Calderón-Zygmund operators for non doubling measures. Math. Ann. 319, 89-149 (2001)

[3] Chen, W., Sawyer, E.: A note on commutators of fractional integral with $R B M O(\mu)$ function. Il. J. Math. 46(4), 1287-1298 (2002)

[4] Sawano, Y., Tanaka, H.: Morrey spaces for non-doubling measures. Acta Math. Sin. English Ser. 21, 1535-1544 (2005)

[5] Sawano, Y., Tanaka, H.: Sharp maximal inequality and commutators on Morrey spaces with non-doubling measures. Taiwanese J. Math. 11(4), 1091-1112 (2007)

[6] Hu, G., Meng, Y., Yang, D.: Multilinear commutators of singular integrals with non-doubling measures. Integr. Equ. Oper. Theory 51, 235-255 (2005)

[7] Sawano, Y.: Generalized Morrey spaces for Non-doubling measures. Nonlinear Diff. Equ. Appl. 15, 413-425 (2008)

[8] Tao, X., Shi, Y., Zhang, S.: Boundedness of multilinear Riesz potential operators on product of Morrey spaces and Herz-Morrey spaces. Acta Math. Sin. Chin. Ser. 52, 535-548 (2009)

X. Tao, T. Zheng

Department of Mathematics, Zhejiang University of Science and Technology, Hangzhou 310023, People's Republic of China

e-mail: xxtao@hotmail.com

T. Zheng

e-mail: taotzheng@126.com

Received: 17 August 2010.

Accepted: 20 December 2010. 